\title{
Potent tumor tropism of induced pluripotent stem cells and induced pluripotent stem cell-derived neural stem cells in the mouse intracerebral glioma model
}

\author{
TOMOHIRO YAMAZOE, SHINICHIRO KOIZUMI, TOMOHIRO YAMASAKI, \\ SHINJI AMANO, TSUTOMU TOKUYAMA and HIROKI NAMBA \\ Department of Neurosurgery, Hamamatsu University School of Medicine, Hamamatsu 431-3192, Japan
}

Received August 1, 2014; Accepted September 12, 2014

DOI: $10.3892 /$ ijo.2014.2702

\begin{abstract}
Although neural and mesenchymal stem cells have been well-known to have a strong glioma tropism, this activity in induced pluripotent stem cells (iPSCs) has not yet been fully studied. In the present study, we tested tumor tropic activity of mouse iPSCs and neural stem cells derived from the iPSC (iPS-NSCs) using in vitro Matrigel invasion chamber assay and in vivo mouse intracranial tumor model. Both iPSC and iPS-NSC had a similar potent in vitro tropism for glioma conditioned media. The migrated iPSCs to the gliomas kept expressing Nanog-GFP gene, suggesting no neuronal or glial differentiation. iPSCs or iPS-NSCs labeled with 5-bromo-2-deoxyuridine were intracranially implanted in the contralateral hemisphere to the GL261 glioma cell implantation in the allogeneic C57BL/6 mouse. Active migration of both stem cells was observed 7 days after implantation. Again, the iPSCs located in the tumor area expressed Nanog-GFP gene, suggesting that the migrated cells were still iPSCs. These findings demonstrated that both iPSCs and iPS-NSCs had potent glioma tropism and could be candidates as vehicles in stem cell-based glioma therapy.
\end{abstract}

\section{Introduction}

Malignant gliomas are the most common primary neoplasms of the central nervous system in adults. The median survival time is about a year and half from diagnosis, despite the modern treatment including surgery, postoperative radiotherapy combined with temozolomide-based chemotherapy $(1,2)$.

Correspondence to: Professor Hiroki Namba, Department of Neurosurgery, Hamamatsu University School of Medicine, 1-20-1 Handayama, Higashi-ku, Hamamatsu 431-3192, Japan

E-mail: hnamba@hama-med.ac.jp

Key words: induced pluripotent stem cells, neural stem cells, glioma, migration, gene therapy
Although improvement of survival time by maximum tumor resection has been reported $(3,4)$, complete surgical removal is not possible because of the invasive nature of gliomas to the surrounding eloquent brain. Residual tumor cells are usually resistant to standard radiotherapy and efficient chemotherapy cannot be delivered because of the presence of the blood-brain barrier and systemic toxicity. Most patients thus die of tumor proliferation after a certain period. To kill those residual tumor cells by increasing the concentration of chemotherapeutic agents in the tumor cells without increasing systemic toxicity, local treatment with biodegradable 1,3-bis (2-chloroethyl)1-nitrosourea wafers (Gliadel wafers) was developed and prolonged survival in patients with recurrent glioblastoma multiforme, the most malignant phenotype of gliomas, was demonstrated (5). However, spread of the drug from implanted Gliadel wafers to the surrounding brain tissues is still limited within millimeter order (6).

In order to expand the treatment area, our previous studies have focused on testing neural stem cells (NSCs) and mesenchymal stem cells (MSCs) that have potent migratory activity toward brain lesions including tumors $(7,8)$. Rat brain tumors were successfully treated by an intratumoral injection of genetically engineered NSCs and MSCs $(9,10)$, and this efficacy was still potent even when the treatment cells were injected at distant intracranial sites from the tumor (11). Therefore, treatment strategies using such stem cells as vehicles for antitumor agents may be promising against malignant glioma because it is a tumor-specific delivery system that spares healthy brain tissue $(12,13)$. However, NSCs and MSCs proliferate relatively slowly and it is not easy to prepare a sufficient amount of cells for treatment.

In 2007, induced pluripotent stem cells (iPSCs) were established. The usefulness of iPSC-based therapies in various fields including regenerative medicine has been investigated $(14,15)$. Because of high proliferative activity of iPSCs, sufficient amount of treatment cells can be prepared. Our previous study demonstrated in vitro migratory activity of mouse iPSCs to glioma-conditioned media and tumorassociated specific growth factors (16). In the present study, in vivo tumor tropic activity of iPSCs and iPSC-derived neural stem-like cells were investigated using a mouse intracranial glioma model. 


\section{Materials and methods}

iPSC and neural induction. The mouse iPSCs, iPS-MEFNg-20D-17 established by Okita et al (15) and transfected with Nanog-GFP as undifferentiation marker, were obtained from Riken BioResource Center (Tsukuba, Japan) and were cultured on mitomycin-treated mouse embryonic fibroblasts feeder cell layers in Dulbecco's modified Eagle's medium (DMEM; Sigma-Aldrich Japan, Tokyo, Japan) high glucose 1X (Invitrogen, Tokyo, Japan) supplemented with $15 \%$ fetal bovine serum (FBS; Sigma-Aldrich Japan), 0.1 mM MEM nonessential amino acids (Invitrogen), $0.1 \mathrm{mM}$ 2-mercaptoethanol (Sigma-Aldrich Japan) and 1,000 U/ml murine leukemia inhibitory factor (mLIF, ESGRO; Millipore, Temecula, CA, USA) according to the protocol previously reported (17). The iPSCs cultured on the gelatin-coated dish without mouse embryonic fibroblasts (passages 10-20) were used in the following experiments. The iPSCs were dissociated at $37^{\circ} \mathrm{C}$ for 2 min using $0.25 \%$ trypsin with $1 \mathrm{mM}$ EDTA.

The adherent monoculture method was used as a differentiation multistep protocol to generate neural stem-like cells from iPSCs $(18,19)$. The mouse iPSCs were dissociated and plated onto $0.1 \%$ gelatin-coated $100-\mathrm{mm}$ dish at a density of $1.2 \times 10^{6} /$ dish and cultured in N2B27 medium that contained a 1:1 mixture of DMEM/F12 supplemented with $1 \% \mathrm{~N} 2$ (Invitrogen) and neurobasal medium supplemented with $2 \%$ B27 (Invitrogen). Medium was renewed every day. Cells were maintained for 12 days and then were dissociated and plated on $0.1 \%$ gelatin-coated $100-\mathrm{mm}$ dish in NSC expansion medium consisting DMEM/F12 medium supplemented with GlutaMAX (Invitrogen), 2\% B27, $50 \mathrm{U} / \mathrm{ml}$ penicillin, $50 \mu \mathrm{g} / \mathrm{ml}$ streptomycin, $20 \mathrm{ng} / \mathrm{ml}$ basic fibroblast growth factor (Sigma-Aldrich Japan) and $20 \mathrm{ng} / \mathrm{ml}$ epidermal growth factor (Invitrogen). Half of the cell-culture medium was changed every 2 days and cells were split at the ratio of 1:2 when reached 90\% confluence using Accutase (Sigma-Aldrich Japan). In the following experiment, the iPSC-derived neural stem-like cells during 10-20 passages were used (named as iPS-NSCs).

Mouse glioma sphere cells. The mouse glioma cell line GL261 and the rat glioma cell line C6 were purchased from the Health Science Research Resources Bank (Osaka, Japan), and the human glioma cell lines U87 from the American Type Culture Collection (ATCC, Manassas, VA, USA). The cells were grown in DMEM supplemented with $10 \%$ FBS, penicillin $(100 \mathrm{IU} / \mathrm{ml})$ and streptomycin $(100 \mu \mathrm{g} / \mathrm{ml})$ at $37^{\circ} \mathrm{C}$ in a humidified atmosphere of $5 \% \mathrm{CO}_{2}$. The glioma cell lines were dissociated using $0.25 \%$ trypsin with $1 \mathrm{mM}$ EDTA for $3 \mathrm{~min}$.

For establishment of GL261 sphere cells $(20,21)$, GL261 cells were seeded in 24-well plates at $2 \times 10^{4}$ cells/well (BD Falcon) containing $500 \mu 1$ DMEM, supplemented with $10 \%$ FBS, $100 \mathrm{U} / \mathrm{ml}$ penicillin $\mathrm{G}$ and $100 \mu \mathrm{g} / \mathrm{ml}$ streptomycin for around $18 \mathrm{~h}$. Culture medium $(250 \mu \mathrm{l})$ was replaced with an equal volume of NSC expansion medium with $1,000 \mathrm{U} / \mathrm{ml} \mathrm{mLIF}$ and vincristine ( $5 \mathrm{ng} / \mathrm{ml}$; Wako). This procedure was repeated every 24 h until day 7 . Then all culture medium was discarded and the wells were filled with $1 \mathrm{ml}$ fresh NSC expansion medium with $1,000 \mathrm{U} / \mathrm{ml} \mathrm{mLIF}$ (without vincristine) on day 7 and changed every 3 days. On day 15 after filled with NSC expansion medium with $1,000 \mathrm{U} / \mathrm{ml} \mathrm{mLIF}$, the spheres were dissociated and single cells were seeded in 96-well plates in $0.1 \mathrm{ml} /$ well of serum-free NSC medium. Every 3 days, $0.02 \mathrm{ml}$ NSC medium was added to the culture. The cells from the well containing spheres with $>50$ cells were dissociated and cultured for expansion in NSC expansion medium with $1,000 \mathrm{U} / \mathrm{ml} \mathrm{mLIF}$.

Migration of iPSCs and iPS-NSCs towards the glioma-conditioned media. The in vitro migratory capacity of iPSCs was assessed using the 24-well Matrigel invasion chamber (BD Biosciences Discovery Labware, Bedford, MA, USA), which contained an $8-\mu \mathrm{m}$ pore size PET membrane treated with Matrigel basement membrane matrix in the insert (16). First, $0.5 \mathrm{ml}$ DMEM was added to the interior of the inserts and the bottom of the wells and allowed to rehydrate for $2 \mathrm{~h}$ at $37^{\circ} \mathrm{C}$ in a $5 \% \mathrm{CO}_{2}$ humidified atmosphere after the gelatin coated both membranes. The DMEM was then carefully removed without disturbing the layer of Matrigel matrix on the membrane. The mouse iPSCs were washed twice in phosphate-buffered saline (PBS) and resuspended to $2 \times 10^{6}$ cells $/ \mathrm{ml}$. The cell suspension $(0.5 \mathrm{ml})\left(1 \times 10^{6}\right.$ cells) was then added to the upper insert. The lower chamber was filled with $0.75 \mathrm{ml}$ of conditioned medium (CM) of the glioma cell lines as well as unconditioned DMEM as a control. CM was obtained by collecting, centrifuging and filtering medium from GL261, C6 and U87 clones $\left(1 \times 10^{6}\right)$, which were cultured in $10 \mathrm{ml}$ of DMEM without FBS for $48 \mathrm{~h}$. Following incubation of Matrigel invasion chambers for $24 \mathrm{~h}$ at $37^{\circ} \mathrm{C}$ in a $5 \% \mathrm{CO}_{2}$ humidified atmosphere, the non-invading cells and/or Matrigel matrix were removed from the upper surface of the membrane in the inserts with a cotton swab. The cells migrating to the lower surface of the membrane were stained with Diff-Quick kit (International Reagents, Hyogo, Japan), which was achieved by sequentially transferring the inserts to air dry. The nuclei of migrated cells were counted in 4 high-power fields (HPF) per membrane with x200 magnification. The images of Nanog-GFP expression in the migrated cell were acquired with EVOS fluorescence microscope (LMS Co., Ltd., Tokyo, Japan). All experiments were conducted in triplicate and results were expressed as the mean number of cells migrating per field \pm SD.

Immunofluorescence for characterization of iPS-NSC. Cultured cells were fixed with $4 \%$ paraformaldehyde for $15 \mathrm{~min}$ at room temperature. For labeling intracellular antigens, cells were permeabilized in PBS containing $0.5 \%$ Triton X-100. After washing in PBS, cells were blocked with 5\% FBS in PBS for $1 \mathrm{~h}$ and incubated overnight in 3\% FBS in PBS with following primary antibodies: Oct4 (1:100; Santa Cruz Biotechnology), Sox2 (1:100; Chemicon), Pax6 (1:100; MBL Eppendorf) and Nestin (1:200; Abcam). After two washes in PBS, appropriate secondary antibodies conjugated to Alexa Fluorophores 488 or 568 (Invitrogen) were diluted at 1:500 in blocking solution and mixed with DAPI ( $1 \mu \mathrm{g} / \mathrm{ml}$; Invitrogen) to counterstain the nuclei. The cells were then washed twice in PBS. Images were acquired with an EVOS microscope.

In vivo migration assay using 5-bromo-2-deoxyuridine (BrdU)-labeled iPSCs and iPS-NSCs. The following experiments were performed according to the Rules of Animal Experimentation and the Guide for the Care and Use of 

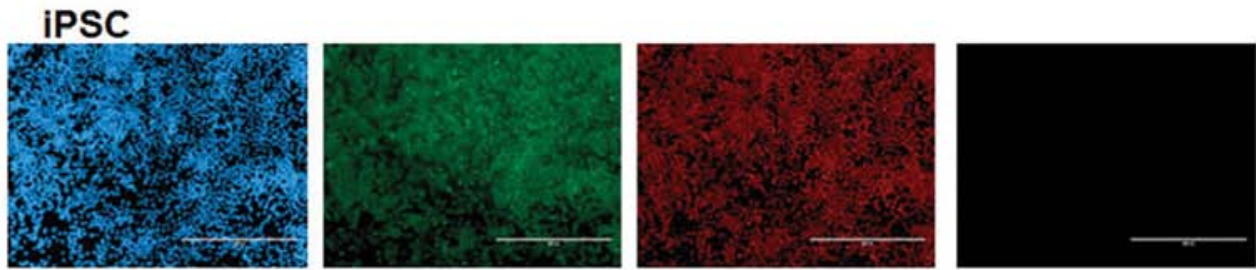

iPS-NSC
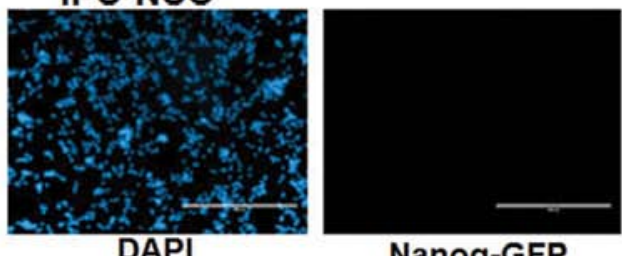

Nanog-GFP

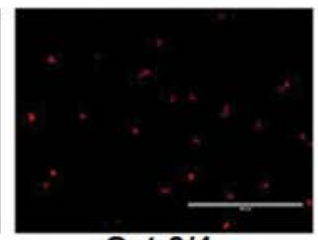

Oct $3 / 4$

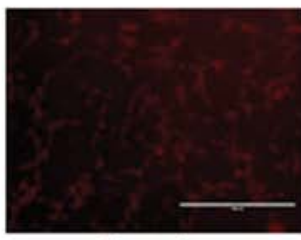

Pax-6

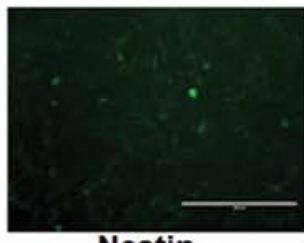

Nestin

Figure 1. Characterization of mouse iPSCs expressing Nanog-GFP and iPS-NSCs. Immunocytochemical staining of iPSCs shows pluripotent stem cell marker, Oct3/4 as well as Nanog-GFP and that of iPS-NSCs shows neural stem cell markers, Pax6 and nestin (bar, $400 \mu \mathrm{m}$ ).

Laboratory Animals of Hamamatsu University School of Medicine and approved by the Institutional Animal Care and Use Committee of Hamamatsu University School of Medicine (H23-012-2011031).

For the in vivo transplantation experiments (22), iPSCs or iPS-NSCs cultured for 5 days were pulsed for $48 \mathrm{~h}$ with $5 \mu \mathrm{M}$ BrdU (Sigma) in each appropriate medium before harvest. iPSCs were harvested by incubation with $0.25 \%$ trypsin for $3 \mathrm{~min}$ and iPS-NSCs, with Accutase for $5 \mathrm{~min}$ at room temperature followed by gentle scraping and resuspended in PBS for implantation.

Six male C57BL/6 mice (19-21 g, 6-weeks old; Nippon SLC, Hamamatsu, Japan) were anesthetized with $0.4 \mathrm{ml} / 100 \mathrm{~g}$ equithesin and placed in a stereotaxic apparatus (Narishige Scientific Instrument Laboratory, Tokyo, Japan). The bregma and lambda were placed in the same horizontal plane. The scalp was incised at the midline and retracted laterally to make a burr hole at an appropriate location (coordinates with respect to bregma: $1 \mathrm{~mm}$ posterior, $2 \mathrm{~mm}$ right). A 25-gauge needle was inserted to the point of $4 \mathrm{~mm}$ ventral from the dura, left in position for $1 \mathrm{~min}$, and then withdrawn to the point of $3 \mathrm{~mm}$ ventral from the dura, where $1 \times 10^{4}$ GL261 sphere cells per $5 \mu$ PBS were infused with a $50 \mu 1$ microsyringe (Hamilton Company, Reno, NV, USA) and a microinjector (Harvard Apparatus Inc., South Natick, MA, USA) for $5 \mathrm{~min}$. The needle was kept in the point of $2 \mathrm{~mm}$ ventral from the dura for an additional minute before removal. After that, a contralateral burr hole (bregma: $1 \mathrm{~mm}$ posterior, $2 \mathrm{~mm}$ left) was made and $1 \times 10^{5}$ BrdU-labeled iPSCs $(n=3)$ or iPS-NSCs $(n=3)$ per $5 \mu 1$ were injected as stated above. The animals were kept under the same laboratory conditions. All the animals were euthanized by injection of high dose of pentobarbital on day 7 after the implantation and the removed brains were fixed with $4 \%$ paraformaldehyde for $2 \mathrm{~h}$. After the fixation, the brains were kept in $20 \%$ sucrose $4^{\circ} \mathrm{C}$ overnight then blocked in OCT embedding compound and snap-frozen at $-78^{\circ} \mathrm{C}$ using Histo-Tek PINO (Sakura Finetek Japan Co., Ltd., Tokyo, Japan). Serial coronal cryosections $(10 \mu \mathrm{m})$ were obtained and stained with hematoxylin and eosin. Sister sections were prepared for dual-filter immunofluorescence.
For the immunofluorescence (23), these sections were treated with citric acid at $>97^{\circ} \mathrm{C}$ for $15 \mathrm{~min}$ and allowed to cool at room temperature for $1 \mathrm{~h}$ to denature DNA. After washing in PBS, the sections were blocked with $1 \%$ bovine serum albumin (BSA) in PBS for 15 min and incubated overnight in $1 \%$ BSA in PBS with anti-BrdU antibody (1:100; Sigma) at $4^{\circ} \mathrm{C}$. For the conformation of GFP (Nanog) expression, the sections of mice injected with iPSCs were additionally stained with anti-GFP antibody conjugated to Alexa Fluorophores 488 (1:500; Molecular Probes-Invitrogen). After two washes in PBS, appropriate secondary antibody conjugated to Alexa Fluorophores 568 (1:500; Molecular Probes-Invitrogen) and DAPI (1 $\mu \mathrm{g} / \mathrm{ml}$; Molecular Probes-Invitrogen) in blocking solution was applied for $1 \mathrm{~h}$ at room temperature. The sections were examined and photographed under an FV1000 fluorescent microscope.

Statistical analysis. The data (means \pm SD) were analyzed using the two-tailed unpaired Student's t-test with $95 \%$ confidence interval for a two-group comparison. Differences were considered significant at $\mathrm{P}<0.05$.

\section{Results}

Characterization of $i P S C$ and $i P S-N S C$. Immunocytochemical characterization of iPS-NSCs was Pax6 (+), nestin (+) and Oct3/4 (-), Nanog-GFP (-), while that of iPSCs was positive for Oct3/4 (+), Nanog-GFP (+) and Pax 6 (-) (Fig. 1).

Migration of iPSCs and iPS-NSCs towards the glioma-CM in vitro. In vitro migratory capacity of iPSCs and iPS-NSCs toward the CM prepared from 3 glioma cell lines (GL261, C6 and U87) was analyzed using the 24-well Matrigel invasion chamber. Significantly higher numbers of iPSCs and iPSNSCs were migrated toward not only mouse GL261-CM but also rat C6-CM and human U87-CM, whereas only a few cells toward the unconditioned medium (Fig. 2A). Representative photomicrographs of the micropore membranes are shown (Fig. 2B, left panels). Most of the migrated iPSCs were positive for Nanog-GFP, suggesting that the migrated iPSCs were not differentiated (Fig. 2B, right panels). 
A

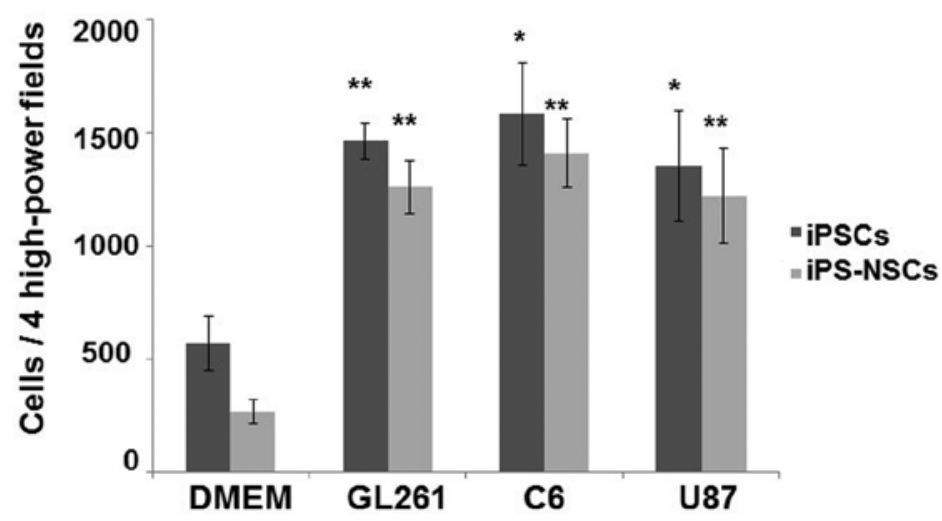

B
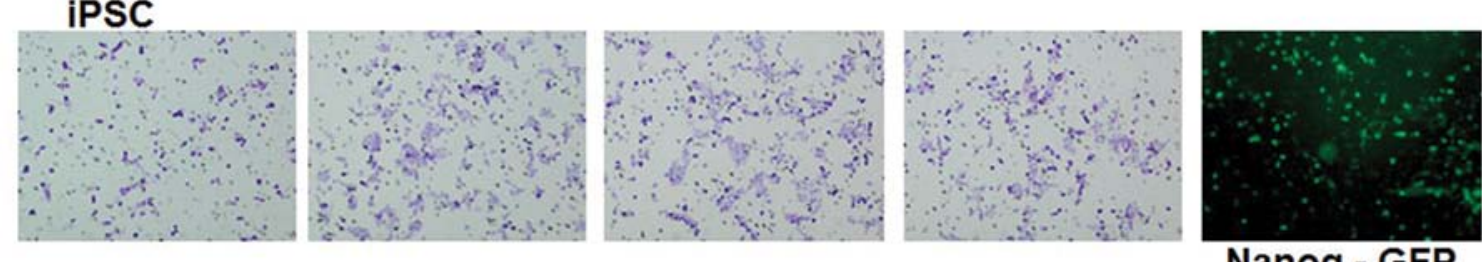

iPS-NSC

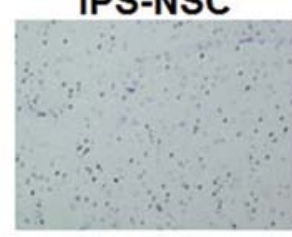

DMEM

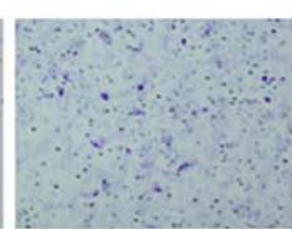

GL261

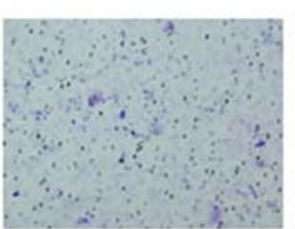

C6

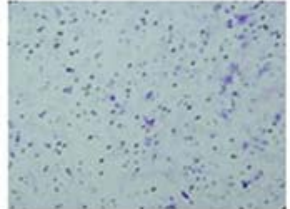

U87

Figure 2. In vitro migration of the mouse iPSCs and iPS-NSCs induced by CM prepared from three rodent (GL261 and C6) and human (U87) glioma cell lines and unconditioned medium in a 24-well Matrigel invasion chamber. (A) Significant migratory capacity of both iPSCs and iPS-NSCs toward glioma $\mathrm{CM}$ compared with unconditioned medium was observed (triplicate, means $\pm \mathrm{SD} ;{ }^{*} \mathrm{P}<0.05,{ }^{* *} \mathrm{P}<0.01$ ). (B) Representative photomicrographs of the micropore membranes from DMEM, GL261-CM, C6-CM and U87-CM. The migrated iPSCs expressed GFP (Nanog).

Migration of iPSCs and iPS-NSCs in the mouse glioma model. BrdU-labeled iPSCs or iPS-NSCs were implanted into the C57BL/6 mouse brain contralateral to the GL261 sphere cell implantation ( $\mathrm{n}=3$ for each group) to assess in vivo migratory capability of iPSCs and iPS-NSCs. Substantial amount of BrdU-labeled iPSCs was observed in the GL261 tumor area, most of which were positive for Nanog-GFP, suggesting that the migrated iPSCs were undifferentiated (Fig. 3). When the iPS-NSCs were used, a similar migratory activity was observed (Fig. 4).

\section{Discussion}

NSCs and MSCs are noteworthy alternative therapeutic tools for malignant glioma because these cells have a strong glioma tropism and immunosuppressive properties to ensure long time reservoirs of therapeutic agents at the tumor sites. Since Aboody and colleagues (24) found an extensive tropism of NSCs for intracranial gliomas, several laboratories have investigated these phenomena using various kinds of stemlike cells. Those cells displayed a unique intrinsic capacity to 'home' the tumor site and to migrate along metastatic/invasive tumor borders even far from the initial site of transplantation. In our previous studies, we demonstrated that NSCs and MSCs have a strong glioma tropism as well as a sufficient bystander effect for a suicide gene therapy using the herpes simplex virusthymidine kinase gene and a prodrug, ganciclovir $(9-11,25)$. In 2010, a clinical trial for evaluating the efficacy/safety of the transplantation of human NSCs genetically modified to express another suicide gene, cytosine deaminase, which convert 5-fuorocytosine (prodrug) to 5-fluorouracil (active drug) at the tumor site, was started for malignant glioma patients in California, USA (13). Though NSCs can potentially be obtained from the adult brain, this process is very complicated and timeconsuming. As an alternative, most studies use a stable cell line of immortalized NSCs originally obtained from embryonic stem cells. Use of embryonic stem cells obviously implies a serious ethical concern, which can be circumvented by using iPSCs. In addition, iPSCs can be obtained from the patients themselves. In fact, suicide gene therapies using iPSC-derived NSCs as cellular vehicles have been already studied $(18,26)$.

In our previous study, mouse iPSCs exerted in vitro tropism to glioma CM as well as various growth factors (16). The present in vivo study was designed to examine whether iPSCs had a glioma tropic activity in the brain as in the case of in vitro study. The implanted iPSCs may migrate or stay at the site of implantation. When the implanted iPSCs migrate, the migrated cells may not be iPSCs, because implanted iPSCs may easily change to more differentiated forms. Our findings demonstrated that the intracranially implanted iPSCs 


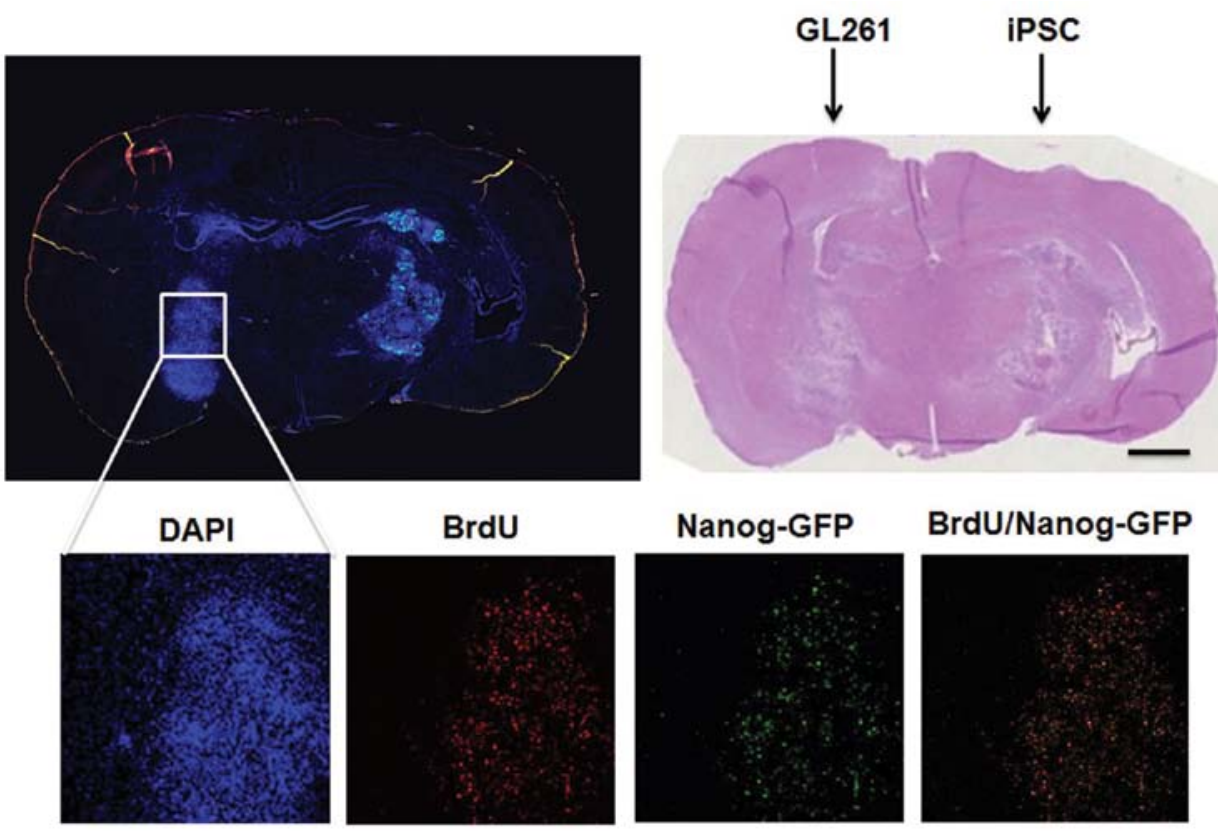

Figure 3. In vivo migration of the BrdU-labeled mouse iPSCs in the intracranial GL261 glioma model. Top panels: overview of the brain coronal sections after hematoxylin and eosin staining (right) and immunofluorescence staining (left). Bottom panels: numerous BrdU-labeled cells were observed in the tumor site (red) and those cells expressed GFP (Nanog) (green), suggesting that the migrated iPSCs were undifferentiated.

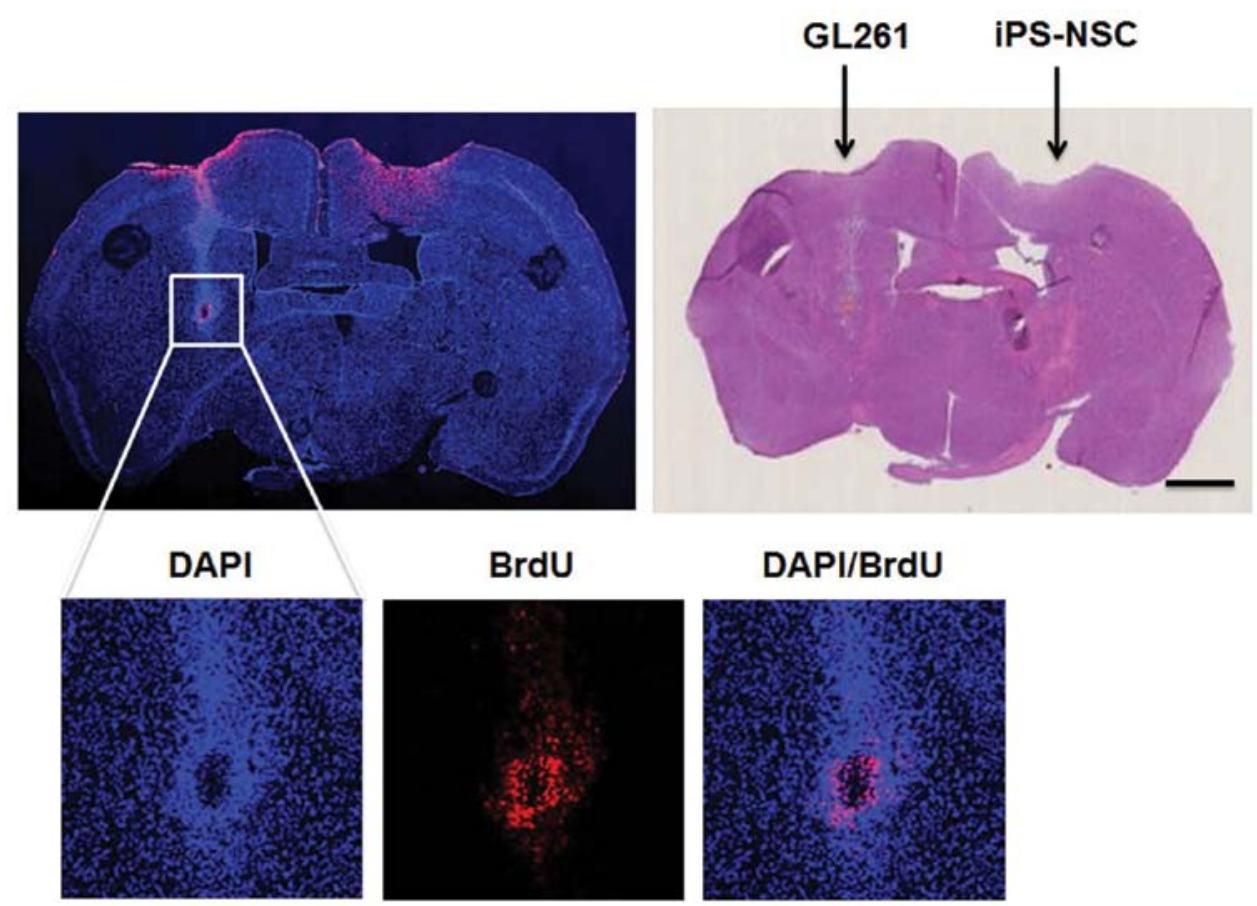

Figure 4. In vivo migration of the BrdU-labeled mouse iPS-NSCs in the intracranial GL261 glioma model. Top panels: overview of the brain coronal sections after hematoxylin and eosin staining (right) and immunofluorescence staining (left). Bottom panels: numerous BrdU-labeled cells were observed in the tumor site.

migrated to the tumor site in the contralateral hemisphere. Interestingly, most of the migrated cells were still iPSCs, which was confirmed by positive staining for GFP (Nanog), a marker for iPSC.

Because iPSCs are known to form teratomas when implanted in the brain (27). Safety issues are thus more important than efficacy of the treatment in clinical appli- cation of iPSCs. We then tested migratory activity of the safer iPS-NSCs which were transduced from iPSCs after neural induction. Our in vitro study demonstrated that iPS-NSCs displayed a potent migratory activity similar to that of iPSCs and the migrated iPS-NSCs were negative for GFP (Nanog) (data not shown), suggesting no substantial contamination of iPSCs in the prepared iPS-NSCs fraction. 
Our in vivo study also demonstrated a potent tropism of GFP (Nanog)-negative iPS-NSCs to the glioma site in the contralateral hemisphere.

Since the potent migratory activity of iPS-NSCs was similar to iPSCs, use of genetically engineered iPS-NSCs might be a safer strategy for treating the tumor recurrence as a glioma gene therapy. Namely, iPSCs are obtained from the patients themselves, transduced with therapeutic genes and expanded. Those genetically engineered iPSCs are intratumorally implanted after neural differentiation (iPS-NSCs) at the time of tumor recurrence. Alternatively, established allogeneic iPS-NSC lines transduced with therapeutic genes may also be useful, because rejection of the treatment cells after a certain period may not disturb treatment efficacy in the case of cancer gene therapies. In this setting, the treatment strategy can be also applied at the time of the initial surgery. One of the limitations of the present in vivo study is lack of quantitative evaluation of migrated cells. Now, we are developing an in vivo method for semi-quantitative evaluation of 'tumor homing therapeutic cells' using radioactive tracers (28).

In conclusion, the present study demonstrated that both iPSCs and iPS-NSCs present a similarly potent tumor tropism under both in vivo and in vitro conditions. In addition, the iPSCs did not differentiate during migration, since they were positive for GFP (Nanog) in the tumor site. To the best of our knowledge, the present study is the first report to explore the biological behavior of iPSCs after being transplanted. These findings suggest that iPSCs and their derivatives can be feasible candidates as vehicles in stem cell-based gene therapy for the patients with malignant glioma.

\section{Acknowledgements}

The present study was supported by the grants from the Japan Society for the Promotion of Science (grant-in-aid for Scientific Research [B; General], \#25293306 and [C; General], \#23592119).

\section{References}

1. Grossman SA, Ye X, Piantadosi S, et al: Survival of patients with newly diagnosed glioblastoma treated with radiation and temozolomide in research studies in the United States. Clin Cancer Res 16: 2443-2449, 2010.

2. Johnson DR, Ma DJ, Buckner JC and Hammack JE: Conditional probability of long-term survival in glioblastoma: a populationbased analysis. Cancer 118: 5608-5613, 2012.

3. Lacroix M,Abi-Said D, Fourney DR, et al: A multivariate analysis of 416 patients with glioblastoma multiforme: prognosis, extent of resection, and survival. J Neurosurg 95: 190-198, 2001.

4. Sanai N, Polley M, McDermott M, Parsa A and Berger M: An extent of resection threshold for newly diagnosed glioblastomas. J Neurosurg 115: 3-8, 2011.

5. Westphal M, Hilt D, Bortey E, et al: A phase 3 trial of local chemotherapy with biodegradable carmustine (BCNU) wafers (Gliadel wafers) in patients with primary malignant glioma. Neuro Oncol 5: 79-88, 2003.

6. Fleming A and Saltzman W: Pharmacokinetics of the carmustine implant. Clin Pharmacokinet 41: 403-419, 2002.

7. Aboody K, Brown A, Rainov NG, et al: Neural stem cells display extensive tropism for pathology in adult brain: evidence from intracranial gliomas. Proc Natl Acad Sci USA 97: 12846-12851, 2000.
8. Lee J, Elkahloun AG, Messina SA, et al: Cellular and genetic characterization of human adult bone marrow-derived neural stem-like cells: a potential antiglioma cellular vector. Cancer Res 63: 8877-8889, 2003.

9. Amano S, Li S, Gu C, et al: Use of genetically engineered bone marrow-derived mesenchymal stem cells for glioma gene therapy. Int J Oncol 35: 1265-1270, 2009.

10. Li S, Tokuyama T, Yamamoto J, Koide M, Yokota N and Namba H: Bystander effect-mediated gene therapy of gliomas using genetically engineered neural stem cells. Cancer Gene Ther 12: 600-607, 2005.

11. Li S, Gao Y, Tokuyama T, et al: Genetically engineered neural stem cells migrate and suppress glioma cell growth at distant intracranial sites. Cancer Lett 251: 220-227, 2007.

12. Amano S, Gu C, Koizumi S, Tokuyama T and Namba H: Tomoricidal bystander effect in the suicide gene therapy using mesenchymal stem cells does not injure normal brain tissues. Cancer Lett 306: 99-105, 2011.

13. Bovenberg MS, Degeling MH and Tannous BA: Advances in stem cell therapy against gliomas. Trends Mol Med 19: 281-291, 2013.

14. Nishikawa S, Goldstein RA and Nierras CR: The promise of human induced pluripotent stem cells for research and therapy. Nat Rev Mol Cell Biol 9: 725-729, 2008.

15. Okita K, Ichisaka T and Yamanaka S: Generation of germlinecompetent induced pluripotent stem cells. Nature 448: 313-317, 2007.

16. Koizumi S, Gu C, Amano S, et al: Migration of mouse-induced pluripotent stem cells to glioma-conditioned medium is mediated by tumor-associated specific growth factors. Oncol Lett 2: 283-288, 2011.

17. Takahashi K, Okita K, Nakagawa M and Yamanaka S: Induction of pluripotent stem cells from fibroblast cultures. Nat Protoc 2: 3081-3089, 2007

18. Lee E, Lam D, Wu C, et al: Glioma gene therapy using induced pluripotent stem cell derived neural stem cells. Mol Pharm 8: 1515-1524, 2011.

19. Onorati M, Camnasio S, Binetti M, Jung CB, Moretti A and Cattaneo E: Neuropotent self-renewing neural stem (NS) cells derived from mouse induced pluripotent stem (iPS) cells. Mol Cell Neurosci 43: 287-295, 2009.

20. Pellegatta S, Poliani PL, Corno D, et al: Neurospheres enriched in cancer stem-like cells are highly effective in eliciting a dendritic cell-mediated immune response against malignant gliomas. Cancer Res 66: 10247-10252, 2006.

21. Yu SC, Ping YF, Yi L, et al: Isolation and characterization of cancer stem cells from a human glioblastoma cell line U87. Cancer Lett 265: 124-134, 2008.

22. Gao Y, Gu C, Li S, et al: p27 modulates tropism of mesenchymal stem cells toward brain tumors. Exp Ther Med 1: 695-699, 2010.

23. Tang X, Falls DL, Li X, Lane T and Luskin MB: Antigenretrieval procedure for bromodeoxyuridine immunolabeling with concurrent labeling of nuclear DNA and antigens damaged by $\mathrm{HCl}$ pretreatment. J Neurosci 27: 5837-5844, 2007.

24. Aboody K, Najbauer J and Danks M: Stem and progenitor cellmediated tumor selective gene therapy. Gene Ther 15: 739-752, 2008.

25. Li S, Tokuyama T, Yamamoto J, Koide M, Yokota N and Namba H: Potent bystander effect in suicide gene therapy using neural stem cells transduced with herpes simplex virus thymidine kinase gene. Oncology 69: 503-508, 2005.

26. Yang J, Lam DH, Goh SS, et al: Tumor tropism of intravenously injected human-induced pluripotent stem cell-derived neural stem cells and their gene therapy application in a metastatic breast cancer model. Stem Cells 30: 1021-1029, 2012.

27. Kawai H, Yamashita T, Ohta Y, et al: Tridermal tumorigenesis of induced pluripotent stem cells transplanted in ischemic brain. J Cereb Blood Flow Metab 30: 1487-1493, 2010.

28. Pei Z, Lan X, Cheng Z, et al: Multimodality molecular imaging to monitor transplanted stem cells for the treatment of ischemic heart disease. PLoS One 9: e90543, 2014. 